\title{
Entre Armorique et Normandie : lecture spatiale du Néolithique ancien et moyen dans l'Ouest de la France à partir de nouvelles données en Mayenne
}

Between Armoric and Normandy: Spatial Readind of Early and Middle Neolithic in Western France from New Data in Mayenne

\section{Gwenolé Kerdivel}

\section{OpenEdition Journals}

Édition électronique

URL : https://journals.openedition.org/rao/1785

DOI : 10.4000/rao.1785

ISBN : 978-2-7535-2790-4

ISSN : 1775-3732

Éditeur

Presses universitaires de Rennes

Édition imprimée

Date de publication : 30 décembre 2012

Pagination : 89-106

ISBN : 978-2-7535-2641-9

ISSN : 0767-709X

Référence électronique

Gwenolé Kerdivel, «Entre Armorique et Normandie : lecture spatiale du Néolithique ancien et moyen dans l'Ouest de la France à partir de nouvelles données en Mayenne ", Revue archéologique de l'Ouest [En ligne], 29 | 2012, mis en ligne le 30 décembre 2014, consulté le 22 août 2022. URL : http:// journals.openedition.org/rao/1785; DOI : https://doi.org/10.4000/rao.1785

Ce document a été généré automatiquement le 22 août 2022.

Tous droits réservés 


\section{Entre Armorique et Normandie : lecture spatiale du Néolithique ancien et moyen dans l'Ouest de la France à partir de nouvelles données en Mayenne}

Between Armoric and Normandy: Spatial Readind of Early and Middle Neolithic in Western France from New Data in Mayenne

\section{Gwenolé Kerdivel}

\section{NOTE DE L'ÉDITEUR}

Manuscrit proposé le 2 juillet 2011, accepté le 12 mars 2012.

\section{Introduction}

$\mathrm{Au}$ cours d'un récent travail d'archéologie spatiale portant sur le Néolithique à l'interface entre les Massifs armoricain et central et les Bassins parisien et aquitain, en impliquant une base de données de plusieurs milliers de gisements, un état des lieux de la distribution spatiale des gisements néolithiques a été mené (Kerdivel, 2012a). À des zones bien ou assez bien couvertes par la recherche, telle que la Normandie ou le Centre-Ouest, dont témoignent notamment plusieurs ouvrages de synthèse, répondaient des zones où le Néolithique était plutôt méconnu, notamment les anciennes provinces du Maine - Mayenne et Sarthe - et de l'Anjou (Joussaume et Pautreau, 1991; Verron, 2000). Par conséquent, l'attention a été portée sur ces départements et en particulier la Mayenne qui fait l'objet du présent article. En effet, sa position géologique, essentiellement sur le Massif armoricain, et sa position 
géographique d'interface en font un secteur clef pour comprendre l'occupation de l'espace dans l'Ouest de la France (ibid.). Or, le Néolithique de Mayenne est surtout connu par ses monuments mégalithiques, dont les premiers inventaires datent de la charnière $\mathrm{XIX}^{\mathrm{e}}$-Xx $\mathrm{x}^{\mathrm{e}}$ siècles (Le Fizelier, 1878 ; Ledru, 1911 et Gilbert, 1964). Les travaux les plus récents ont, pour leur part, concerné des allées couvertes et des sépultures à entrée latérale (Bouillon, 1984, 1989 et 1998 ; Avignon, 1976 et L'Helgouac'h in Collectif, 1986). Il importait donc d'interroger des données récemment acquises pour les étapes anciennes et moyennes du Néolithique, afin de discuter la place du Néolithique de ce département dans son contexte de l'Ouest de la France et aussi de tester un modèle de peuplement proposé récemment à l'échelle des marges du Massif armoricain.

\section{Les traces d'un Néolithique ancien}

2 Les occupations appartenant au Néolithique ancien Villeneuve-Saint-Germain sont encore rares. Le site du Clos-Henry à Château-Gontier a été découvert à l'occasion d'une fouille préventive menée par l'Inrap sous la direction d'A. Valais (Valais et al., 2008; fig. $1, n^{\circ}$ 2). Elle a permis la mise au jour de nombreuses structures d'époques diverses (protohistoire et haut Moyen Âge), ainsi qu'une fosse néolithique (F 40) située à deux mètres de la limite de décapage. Le site est installé sur un étroit rebord de plateau dominant, à $51 \mathrm{~m} \mathrm{NGF}$, un ru qui se jette dans le ruisseau du Moulinet, affluent de la Mayenne.

Figure 1 : Carte de distribution des gisements de Mayenne évoqués dans le texte ( Figure 1: Distribution map of Neolithic sites of Mayenne, discussed in the text

1 : La Cigotière à Ballée ; 2 : La Pastourière à Ballée ; 3 : le Clos-Henry à Bazougers/Château-Gontier ; 4 : Le Val à Couesmes-Vaucé ; 5 : Le Petit Domaine à Couesmes-Vaucé ; 6 : L'ancienne gare à Ernée ; 7 : La Rouairie à Hardanges ; 8 : Le Bignon à Niort-la-Fontaine/Lassay-les-Châteaux ; 9 : Le Petit Montron à Laval ; 10 : La Haute Cadurie à Loigné-sur-Mayenne ; 11 : Cordouin à Oisseau ; 12 : RuilléFroids-Fond ; 13 : La Moinerie à Saint-Denis-d'Anjou ; 14 : Les Erves à Sainte-Suzanne ; 15 : Beulin à Saint-Germain-le-Guillaume ; 16 : La Bourdais I à Saint-Mars-sur-Colmont. 


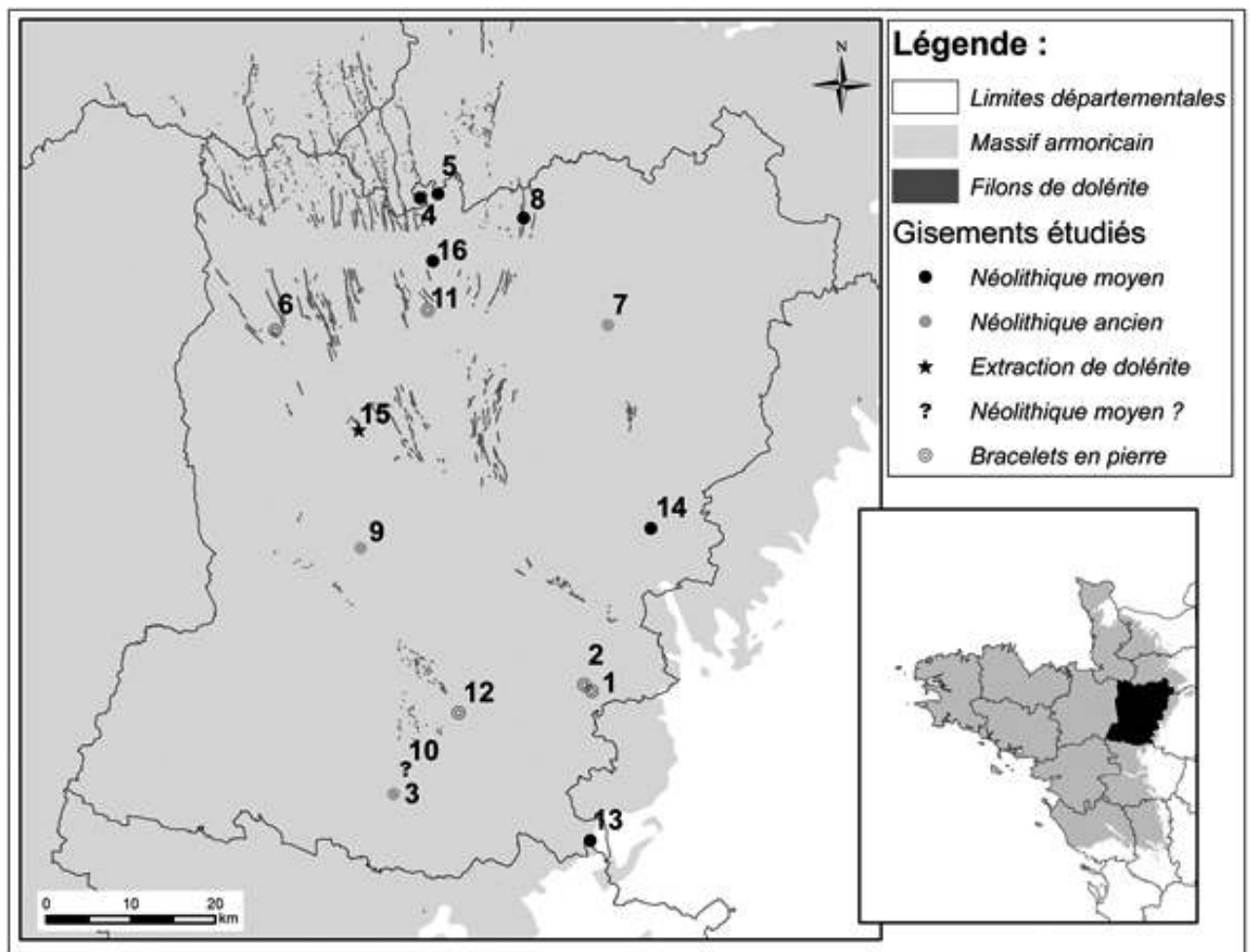

3 Cette fosse, de forme oblongue, mesure trois mètres de long sur 0,50 à 0,80 mètre de large, pour une profondeur de 0,20 mètre. Le fouilleur interprète cette structure comme la fosse d'extraction latérale d'un bâtiment néolithique détruit par l'érosion. Nous y voyons plus volontiers le témoin d'une des fosses satellites de quelques habitats du Villeneuve-Saint-Germain, telles celles reconnues au Haut-Mée (Saint-Étienne-enCoglès, Ille-et-Vilaine ; Cassen et al., 1998). Quoi qu'il en soit, l'habitat classique devait peut-être se situer non loin de là.

4 Cette fosse a livré quelques charbons de bois, 127 tessons et un polissoir à trois faces actives. Cinq individus céramiques ont été individualisés (Valais et al., 2008). Il y aurait trois jarres de grand diamètre, une marmite et une jatte. L'étude des pâtes laisse penser à une provenance locale de l'argile, inférieure à cinq kilomètres du site. Le montage au colombin est soupçonné, les surfaces ont été polies. Les décors sont soit des cordons plastiques soit des boutons ovalaires, fixés au niveau de la lèvre ou en dessous. L'un d'eux porte une anse à ensellement médian. Cette étude céramique attribue avec certitude cette fosse au Villeneuve-Saint-Germain, mais surtout, atteste d'une production céramique locale à cette époque. Un datage radiocarbone est disponible pour cet ensemble : $6115 \pm 60 \mathrm{BP}$ (Lyon-2054), obtenue sur charbons de bois (Valais et al., 2008). Calibré à deux sigmas, le résultat se place au début du $\mathrm{V}^{\mathrm{e}}$ millénaire, correspondant au début du VSG (Dubouloz, 2003).

Le site du Petit Montron à Laval est le résultat de prospections pédestres effectuées par E. Mare ${ }^{1}$ dans les années quatre-vingts (fig. 1, nº 8). Il est situé à l'ouest de Laval, sur un haut versant de vallée à 25 mètres d'un petit ru qui se jette dans le ruisseau des Périls, lui-même affluent de la Mayenne. Parmi le mobilier découvert n'ont été conservés que la moitié d'un bracelet en schiste tacheté ${ }^{2}$ (fig. 2, no 6), un tranchet (fig. 2, n 1), un burin multiple (fig. $2, \mathrm{n}^{\circ} 3$ ), un grattoir (fig. 2, $\mathrm{n}^{\circ} 2$ ), un fragment de lame de hache en silex débité (fig. $2, \mathrm{n}^{\circ} 5$ ) et une probable lame de faucille (fig. $2, \mathrm{n}^{\circ} 4$ ). Cet assemblage lithique, bien que numériquement faible, peut évoquer le Néolithique ancien, puisqu'il 
trouve des éléments de comparaisons avec ceux du Haut Mée à Saint-Étienne-en-Coglès et de Pluvignon à Betton (Ille-et-Vilaine ; Cassen et al., 1998 ; Blanchet et al., 2010).

Figure 2 : Mobilier lithique de surface attribuable pour l'essentiel au Néolithique ancien Figure 2: Lithic artefacts essentially dated from Early Neolithic

1 à 6 : Le Petit Montron à Laval ; 7 : L'ancienne gare à Ernée ; 8 à 10 : Ruillé-Froid-Fonds). Dessin et DAO : G. Kerdivel.

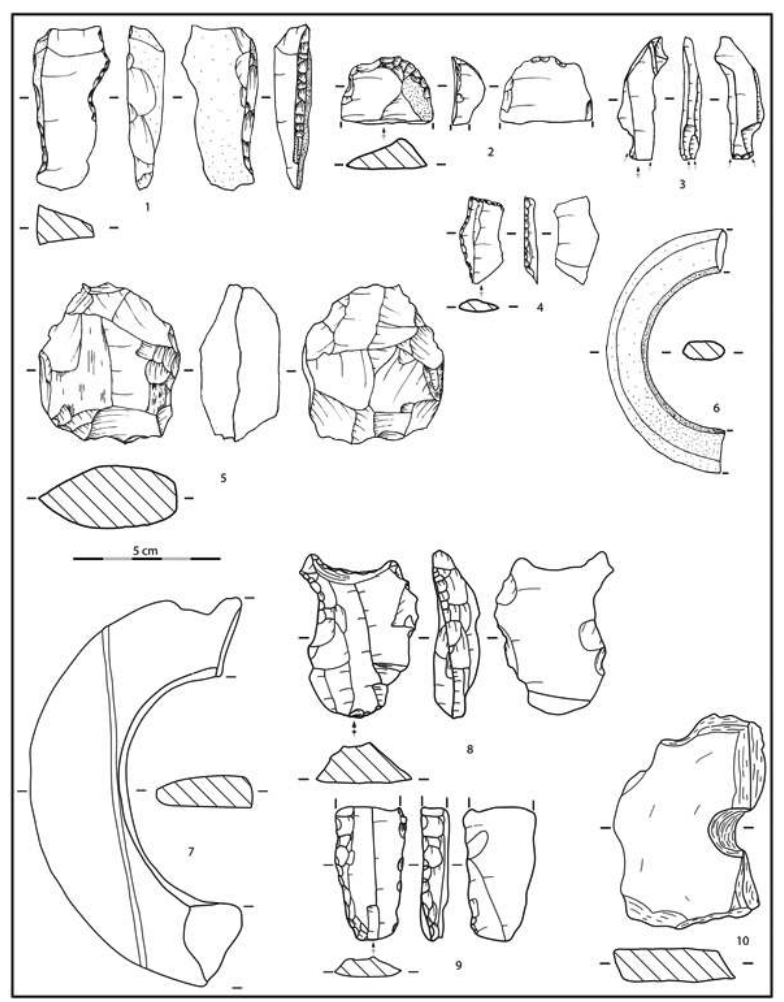

6 Parmi les découvertes isolées et anciennes, un « casse-tête » en silex a été découvert à la fin du XIX ${ }^{e}$ siècle à La Rouairie à Hardanges par M. Montagu (Moreau, 1889; fig. 1, no 6). Disparu, seul un moulage en est conservé au Dépôt archéologique départemental de Jublains. Il se présente sous la forme d'un objet lithique grossièrement circulaire et taillé bifacialement par retouches envahissantes, avec une perforation centrale. On ne peut que déplorer l'impossibilité d'être plus précis dans la description typotechnologique de la pièce et dans son matériau étant donné qu'elle a disparu. Toutefois, ce type de pièce peut être attribuable, à titre d'hypothèse, au VSG récent d'après les travaux de F. Bostyn à Ocquerre (Marne ; Praud 2009).

7 La question des bracelets en schiste et de leur attribution chronologique fait encore débat. En effet, ils sont abondamment représentés au Néolithique ancien de culture Villeneuve-Saint-Germain, mais perdurent aussi au Néolithique moyen dans les cultures cernoïdes (Fromont, 2008).

Plusieurs découvertes de tels objets ont été signalées en Mayenne. La plus ancienne est celle d'un fragment de bracelet en schiste briovérien, découvert dans les déblais de la gare d'Ernée (Moreau, 1880 ; fig. $1, \mathrm{n}^{\circ} 6$ et fig. 2, $\mathrm{n}^{\circ} 7$ ).

Un petit assemblage a été découvert sur la commune de Ruillé-Froid-Fonds, sans plus de précision (fig. $\left.1, \mathrm{n}^{\circ} 12\right)$. Celui-ci est composé d'un perçoir en silex et d'une ébauche en schiste avec perforation centrale (fig. $2, \mathrm{n}^{\circ} 8$ et 10). Ces éléments pourraient être les 
témoins de la technique de perforation par rotation, connue ponctuellement en Normandie pour le façonnage des bracelets (Fromont, 2008). Deux éléments incitent toutefois à la prudence : le fragment de lames de poignard qui accompagnait ces objets (fig. $2, n^{\circ} 9$ ) et la découverte fréquente de ce type de pièces sur les sites protohistoriques (C. Marcigny, communication personnelle). Deux autres découvertes de bracelets en schiste ont été signalées à Ballée sur les sites de la Cigotière et de la Pastourière par P. Lemesle et X. Fournier (fig. $1, \mathrm{n}^{\circ} 1$ et 2 ). Ils sont situés dans la vallée de l'Erve, affluent de la Sarthe, respectivement en fond de vallée et sur un haut versant. La proximité avec l'atelier de façonnage de bracelets des Havardières à Sablé-surSarthe (Sarthe) est à souligner (Bailloud et al., 1987). Par ailleurs, de récentes découvertes de bracelets ont été faites par B. Bodinier le long de la Mayenne et par R. Lerivrain au lieu-dit Cordouin à Oisseau (fig. 1, n 9).

\section{Des données plus nombreuses pour le Néolithique moyen}

\section{Des monuments mégalithiques}

Les découvertes attribuables au Néolithique ancien attestent enfin de cette phase en Mayenne, alors que, pendant longtemps, le plus ancien Néolithique connu des spécialistes dans ce département était à rattacher au "Chasséen", avec le dolmen angevin des Erves à Sainte-Suzanne (fig. 1, n 14 et fig. 3).

Figure 3 : Quelques monuments et leurs mobiliers associés datés du Néolithique moyen. DAO et cliché : G. Kerdivel.

Figure 3: Some monuments and associated artefacts dated from Middle Neolithic.

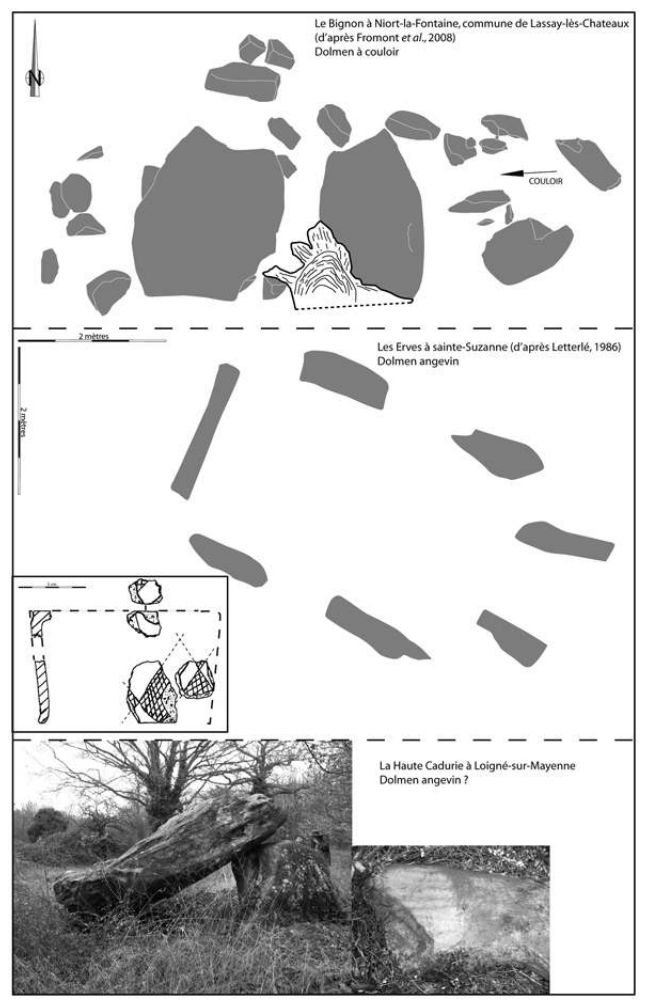


11 Fouillé par F. Letterlé en 1983, le cairn présentait une façade rectiligne et un contour arrondi à l'arrière (Letterlé, 1986). La chambre est de forme quadrangulaire. Elle est formée de cinq dalles mégalithiques non jointives ; l'espace entre ces orthostates était comblé par des parements en pierres sèches. Deux orthostates forment un portique. Malgré quelques différences mineures, ce monument est considéré par M. Gruet comme un dolmen angevin, ce que R. Joussaume appuie (Gruet, 2005 ; Joussaume et al., 2008).

Ce monument a livré les ossements d'au moins cinq individus. Le mobilier céramique (vingt-deux tessons) est notamment composé d'une coupe-à-socle cylindrique à décor de losanges quadrillés, dont les tessons ont été découverts en place, et qui date pour partie l'utilisation du dolmen du Néolithique moyen II. Un datage radiocarbone donne un résultat de $5580 \pm 140 \mathrm{BP}$ (Ly 3100), soit entre 4590 et 4320 avant notre ère (à 1 sigma, et pour $63,7 \%$ de probabilité), avec un écart-type particulièrement large (recalibré à l'aide d'Oxcal). Ce résultat a été obtenu sur un bois de cerf scellé par les pierres de calage d'un orthostate, ce qui permet de dater la construction du monument. Bien qu'apparemment ancien, ce datage renvoie à d'autres obtenus pour des dolmens à couloir de l'Ouest de la France. Ce monument pourrait avoir été occupé pendant une longue période au Néolithique, comme l'atteste un autre datage de $4650 \pm 130 \mathrm{BP}$ (Ly 3099), soit entre 3700 et 3000 avant notre ère (à 2 sigmas), obtenu sur un lot d'os humains dont la provenance stratigraphique est inconnue.

Trop rarement cité dans les inventaires, ce monument est pourtant le premier dolmen angevin fouillé dont l'ancienneté apparaît hautement probable, ce qui a été confirmé par la suite avec les fouilles du dolmen E134 à Taizé et de Puyraveau à Saint-Léger-deMonbrun, (Deux-Sèvres ; Joussaume et Laporte, 2006).

14 J. L'Helgouac'h et R. Bouillon soupçonnent tous deux l'existence d'un autre monument angevin à La Haute-Cadurie à Loigné-sur-Mayenne, bien que ce monument soit très ruiné (L'Helgouac'h in Collectif, 1986 ; fig. $1, n^{\circ} 10$ et fig. 3). À proximité immédiate, il faut signaler un polissoir sur un bloc de grès qui a pu appartenir au monument. J. Rioufreyt suppose aussi un dolmen angevin détruit à La Moinerie à Saint-Denisd'Anjou dans une note adressée au Service régional d'Archéologie et sur la base d'une dalle équarrie ayant pu appartenir à un portique (Kerdivel, 2012a, fig. 1, $n^{\circ} 13$ ).

Plus récemment, un dolmen à couloir a été redécouvert au cours d'une prospection thématique : il s'agit du monument du Bignon à Niort-la-Fontaine, commune de Lassayles-Châteaux (Mayenne, Fromont et al., 2008; fig. 1, $\mathrm{n}^{\circ} 18$ et fig. 7). Sa chambre quadrangulaire ne montre pas d'excavations anciennes et semble bien conservée. Elle est encore couverte d'une table en dolérite, mesurant deux mètres sur un. Un autre bloc, peut-être une autre table, gît sur son flanc oriental. Le couloir dans son état de conservation se développe à l'est, mesure un peu plus de deux mètres et semble légèrement désaxé au nord, permettant de soupçonner un plan en $P$. Morphologiquement, il est très comparable à celui du Creux à Saint-Bômer-les-Forges (Orne ; Chancerel, 1992). 


\section{Des gisements de surface : le Petit Domaine à Couesmes-Vaucé et de la Bourdais I à Saint-Mars-sur-Colmont ${ }^{3}$}

\section{Présentation des gisements}

Le site du Petit Domaine à Couesmes-Vaucé se trouve au nord du département sur un très étroit plateau, culminant à $153 \mathrm{~m}$ NGF (fig. 1 et $4, \mathrm{n}^{\circ}$ 5). Il est installé à proximité de filons de dolérite (Vernhet et al., 1995). Cette collection comporte 909 pièces lithiques, ainsi que quelques tessons de facture préhistorique. À proximité, sur le gisement du Val, quelques objets évoquent aussi un Néolithique moyen lato sensu (fig. 1 et $4, \mathrm{n}^{\circ} 4$ ).

Figure 4 : Carte de situation topographique des sites du Val et du Petit Domaine à Couesmes-Vaucé $\left(\mathrm{n}^{\circ} 4\right.$ et 5$)$, du Bignon à Lassay-les-Châteaux $\left(\mathrm{n}^{\circ} 8\right)$ et de la Bourdais I à Saint-Mars-sur-Colmont (no 16). Dessin et DAO : G. Kerdivel.

Figure 4: Topographical map with sites of Val and Petit Domaine in Couesmes-Vaucé, of Bignon in Lassay-les-Châteaux and of la Bourdais I in Saint-Mars-sur-Colmont.

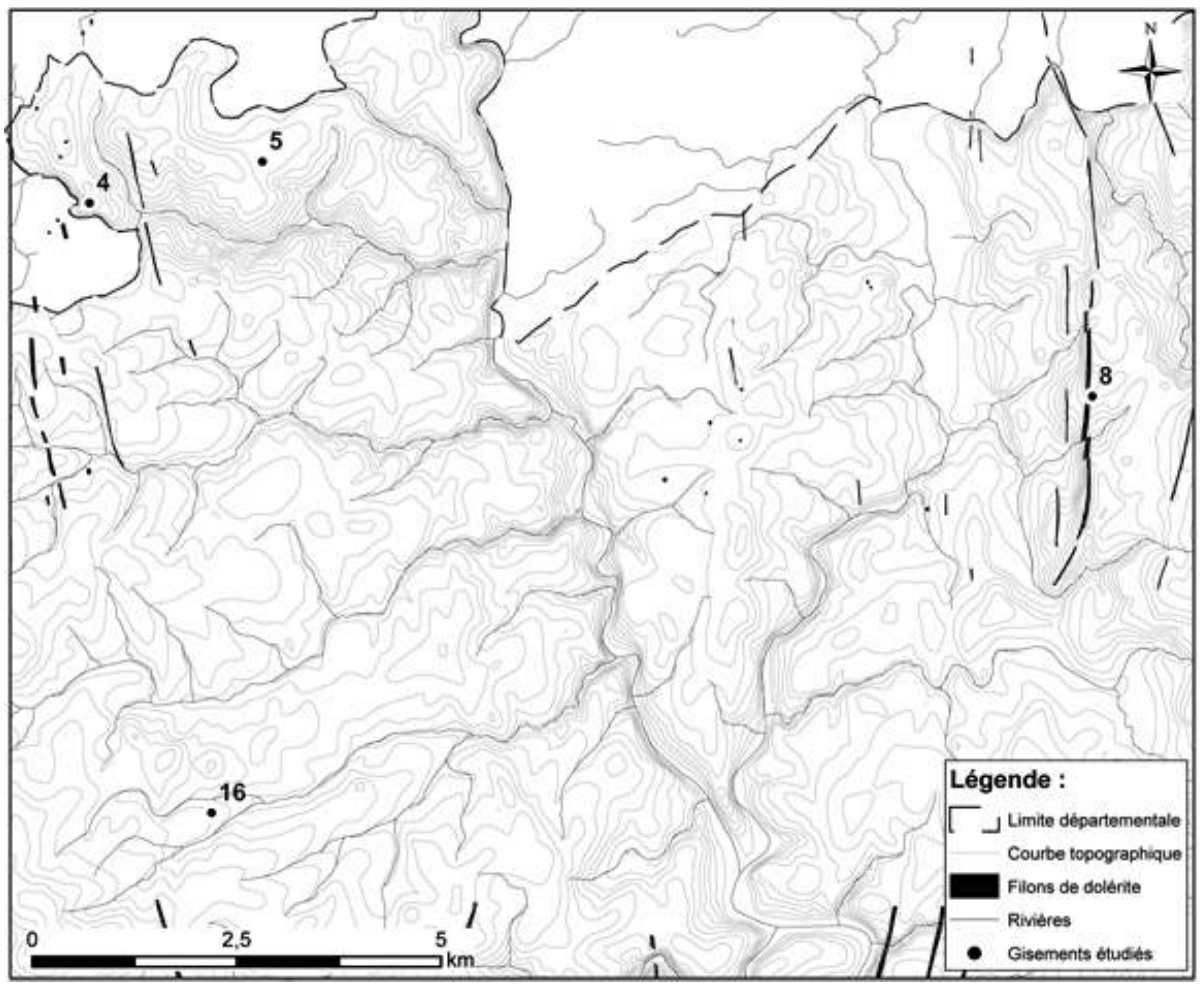

L'industrie lithique est façonnée à près de $97 \%$ en silex. Un peu plus de $25 \%$ de l'ensemble a subi une chauffe plus ou moins importante ne permettant pas de distinguer les couleurs d'origine du silex. Il faut signaler la présence d'un éclat en opale résinite et de deux pièces (éclat et racloir) dans un silex turonien, peut-être de la région du Grand-Pressigny.

Le débitage d'éclats est largement majoritaire (24 nucléus à éclats sur 27 et près de $82 \%$ d'éclats sur l'ensemble des supports non retouchés; fig. $5, n^{\circ} 1$ et 2 ). De même, si l'on observe les supports transformés, les éclats présentant des retouches diverses dominent sur les lames.

Les outils majoritaires sont les grattoirs ( $21 \%$, fig. $5, n^{\circ} 3$ à 7$)$, les coches et denticulés ( $9 \%$ après avoir écarté les coches profondes probablement récentes, fig. $\left.5, n^{\circ} 8\right)$ et les 
tranchets (7 \%, fig. 5, n 9 à 11). Grattoirs et tranchets sont essentiellement façonnés sur éclats. Parmi les autres outils présents, il faut signaler des racloirs (fig. 6, $\mathrm{n}^{\circ} 10$ et 11), des troncatures à retouches abruptes des deux bords (fig. $6, n^{\circ} 7$ et 8 ), des lames ou éclats à bord abattu, quelques fragments de haches polies dont un tranchant (fig. 6, $n^{\circ} 1$ ), trois perçoirs (fig. $6, n^{\circ} 4$ et 9 ), deux burins (fig. $6, n^{\circ} 2$ ) et deux armatures de flèches tranchantes de forme trapézoïdale à retouches abruptes des bords (fig. 6, $\mathrm{n}^{\circ} 5$ et 6). La parure est représentée par un fragment de bracelet en schiste briovérien ${ }^{2}$ perforé et dont ses troncatures ont été polies (fig. 6, n 3 ).

Figure 5 : Mobilier lithique de surface provenant du Petit Domaine à Couesmes-Vaucé $(1$ et 2 : nucléus ; 3 à 7 : grattoirs ; 8 coche ; 3 à 11 : tranchets). Dessin et DAO : G. Kerdivel.

Figure 5: Lithic artefacts from Petit Domaine in Couesmes-Vaucé.

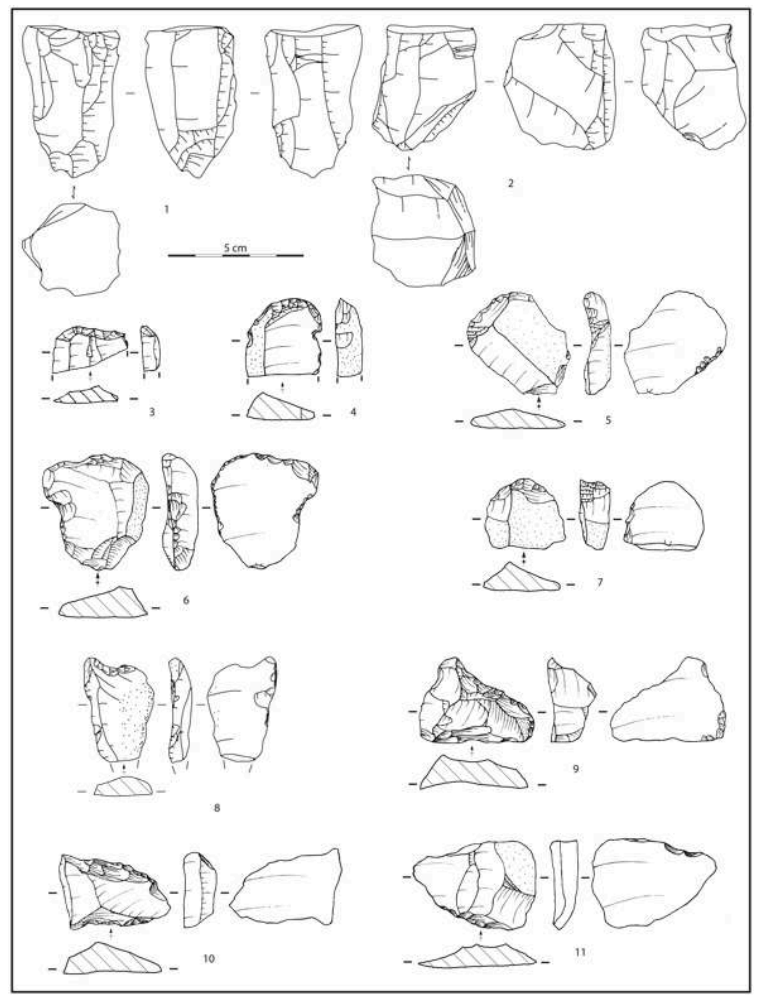

Figure 6 : Mobilier lithique de surface provenant du Petit Domaine à Couesmes-Vaucé : $n^{\circ} 1$ à 11) et de la Bourdais I à Saint-Mars-sur-Colmont

Figure 6: Lithic artefacts from Petit Domaine in Couesmes-Vaucé ( $n^{\circ} 1$ to 11$)$ and from la Bourdais I in Saint-Mars-sur-Colmont ( $n^{\circ} 12$ to 16$)$.

$n^{\circ} 12$ à $16 ; 1$ et 12 : fragments de lames de hache en silex converties en nucléus ; $2:$ burin ; 3 :

fragment d'anneau en schiste perforé ; 4 : perçoirs ; $5,6,13$ et $14:$ armatures de flèches tranchantes ;

7 et 8 : troncatures retouchées (armatures ?) ; 10 : racloir en silex de la région du Grand-Pressigny ;

11 : racloir ; 15 et 16 : fragment et lame entière de hache polie en roche verte. Dessin et DAO : G.

Kerdivel. 


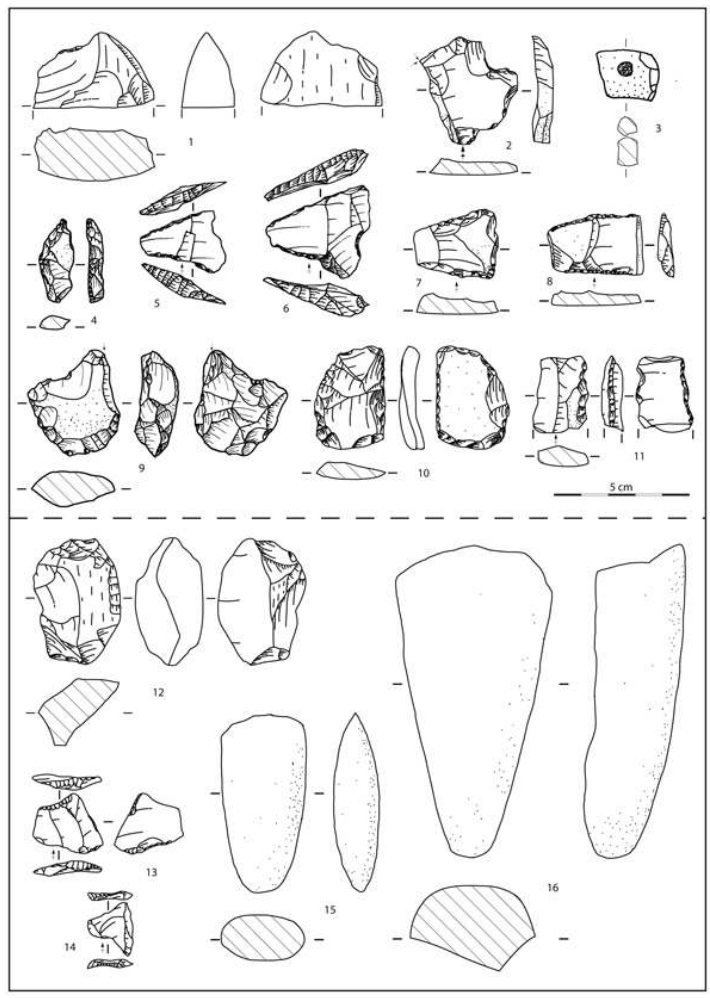

Découvert par R. Lerivrain, le site de la Bourdais I à Saint-Mars-sur-Colmont est situé sur un éperon encadré par deux rus (fig. 1 et $4, \mathrm{n}^{\circ} 16$ ). La collection comprend 301 pièces lithiques et quelques tessons céramiques (fig. 8).

21 L'industrie lithique est façonnée à près de $95 \%$ dans deux variétés de silex, parfaitement comparables à ceux que l'on rencontre au Petit Domaine. Ici, près de $15 \%$ des pièces lithiques ont subi une chauffe plus ou moins importante. Parmi l'outillage, c'est le cas des deux seules armatures de flèches tranchantes à retouches abruptes des bords (fig. $6, \mathrm{n}^{\circ} 13$ et 14). Concernant les autres matières premières, il faut signaler l'existence d'un nucléus et d'un éclat en opale résinite, d'un talon de hache polie en grès (fig. $6, \mathrm{n}^{\circ} 16$ ), d'une hache polie en dolérite (fig. $6, \mathrm{n}^{\circ} 15$ ), de deux fragments de meules en granite et d'un fragment de polissoir en quartz (fig. $7, \mathrm{n}^{\circ} 10$ ).

Le débitage est largement orienté vers la production d'éclats ( $61,5 \%$ des supports non retouchés; fig. $7, \mathrm{n}^{\circ} 1$ ). D'ailleurs, aucun nucléus à lames n'est présent dans la collection; seul le nucléus en opale résinite a servi à tirer des lamelles. De même, les éclats dominent parmi les supports retouchés.

L'outillage en silex est largement dominé par les grattoirs, façonnés essentiellement sur éclat ( $38,5 \%$; fig. $7, n^{\circ} 2$ à 5$)$. Ensuite, on retrouve les coches et denticulés (15\%), ainsi que les tranchets $\left(10 \%\right.$; fig. $7, n^{\circ} 6$ à 8$)$. Autrement, il faut signaler quelques lames à deux bords abattus, deux racloirs, deux armatures de flèches tranchantes trapézoïdales (fig. $6, n^{\circ} 13$ et 14), deux lames à bord abattu, deux retouchoirs, une troncature, un perçoir et un burin (fig. 7, $n^{\circ}$ 9). 
Figure 7 : Mobilier lithique de surface provenant de la Bourdais I à Saint-Mars-sur-Colmont (1 : nucléus ; 2 à 5 : grattoirs ; 6 à 8 : tranchets; 9 : burin ; 10 : polissoir).

Figure 7: Lithic artefacts from la Bourdais I in Saint-Mars-sur-Colmont.

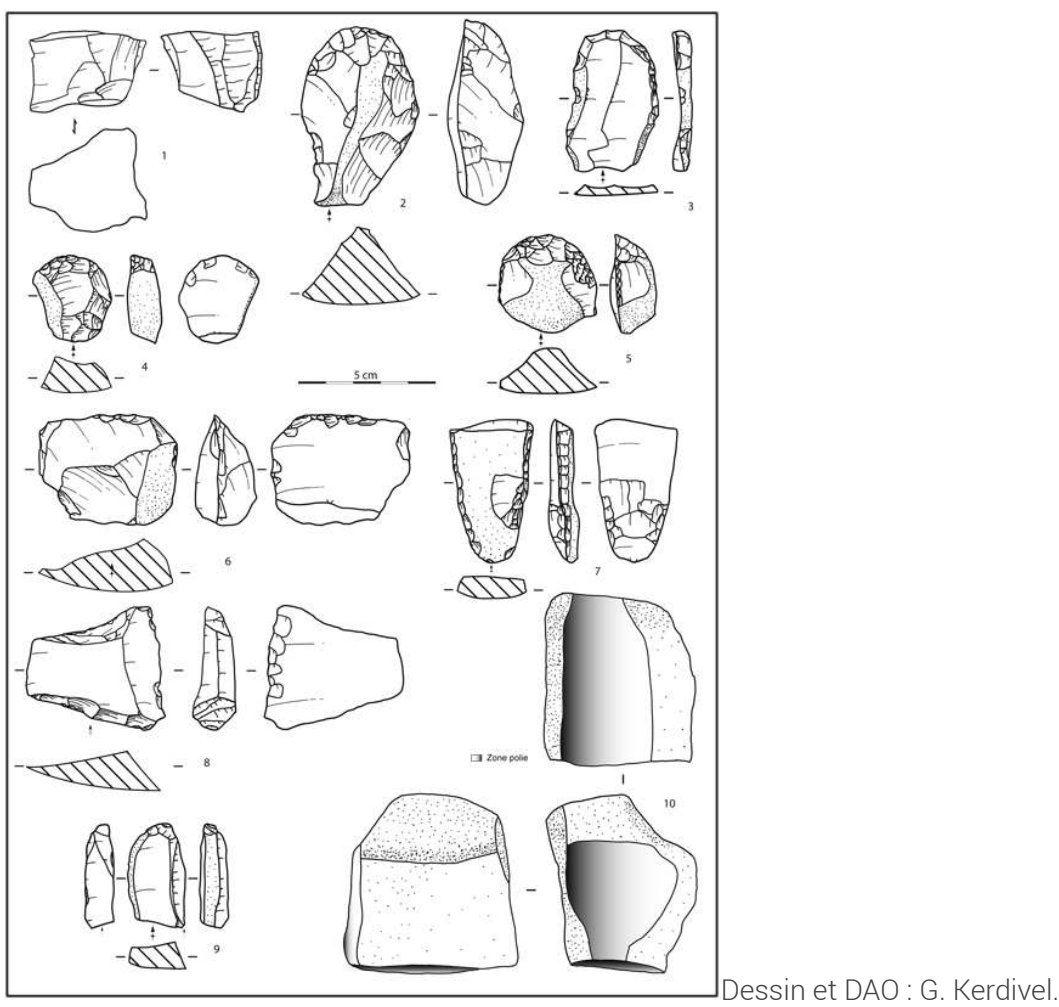

\section{Développement}

Aspect particulier de ces sites, leur industrie lithique y est façonnée à plus de $95 \%$ dans un silex pour lequel deux grands types de provenance sont envisageables (Lasseur, communication orale, Lasseur et al., 2005). La première se caractérise par des silex au cortex très fin, tranchant nettement avec la silice et qui présentent souvent de petites zonations et une patine de couleur rouille. Leurs couleurs varient du gris tacheté au brun, en passant par le bleu. Ces matériaux proviennent d'argiles à silex, que l'on peut rencontrer notamment sur les sommets de versants. Leur exploitation ne pose pas de difficultés particulières : elle a, par exemple, pu se faire en fosses ouvertes comme on en connait à Vion (Sarthe) ou à Ri (Orne ; Georges et al., 2012 ; Marcigny et al., 2011). La deuxième variété de silex est caractérisée par des teintes noir clair à bleu foncé. Elle pourrait provenir de véritables carrières ou minières. Quelle que soit la méthode d'acquisition, la région d'importation de ces silex reste celle d'Écouché (Orne ; Lasseur et al., 2005).

L'approvisionnement en matières premières de ces deux gisements exclut une datation au Néolithique ancien, car si ce n'est la présence abondante de roches siliceuses, aucune caractéristique des industries lithiques du Néolithique ancien n'est représentée (Cassen et al., 1998 et Blanchet et al., 2010). De même, une datation aux phases finales du Néolithique nous paraît à exclure en raison d'un emploi majoritaire du silex, phénomène contraire à ce que l'on observe par ailleurs sur les gisements domestiques de cette phase sur le Massif armoricain (Guyodo, 2003). Plaidant pour une telle attribution, la présence de silex de la région du Grand-Pressigny n'est guère 
discriminante, puisque la diffusion de ce silex semble se mettre en place progressivement dès le Néolithique moyen voire avant, comme l'attestent certains sites proches comme Auzay (Vendée), Angers (Maine-et-Loire) ou Quimper (Finistère, Large et Birocheau, 2004; Marcigny et al., 2007; Tinevez et al., 2006). Le seul racloir à la Bourdais dans ce silex ne permet donc pas de dater le site de la fin du Néolithique.

La production d'éclats à la percussion directe dure majoritaire, telle qu'elle domine dans nos assemblages, se rencontre dès les assemblages Cerny et Chambon dans tout le Nord de la France et va se prolonger jusqu'à la fin du Néolithique (Guyodo, 2005 ; Ghesquière et al., 1998). Les collections de référence pour le Néolithique permettant de comparer le mobilier de ces deux sites restent peu publiées. Au sein de l'outillage des sites mayennais, les éléments les plus caractéristiques sont finalement la présence anecdotique des burins, la forme des armatures de flèches et la présence de tranchets ; les autres outils s'avérant eux largement ubiquistes. Or, au Néolithique moyen I, les burins sont une composante essentielle des assemblages lithiques, représentant par exemple 27 \% au Parc à Vivoin (Sarthe ; Ghesquière et Marcigny, 2003). Par la suite, ils tendent largement à disparaître, comme c'est le cas par exemple à Argentan (Orne ; Ghesquière et Marcigny, 2004) ou à Lillemer (Ille-et-Vilaine; Guyodo 2001b). Leur quasi-absence au Petit Domaine et à La Bourdais oriente la datation au Néolithique moyen II. Or, justement, les armatures de flèches tranchantes trapézoïdales se rencontrent en général plus fréquemment dans les assemblages attribués à cette étape du Néolithique moyen, comme on le voit à Lillemer ou à Auzay (Guyodo, 2001b et Large et Birocheau, 2004). Les tranchets sont véritablement une originalité de ces sites. En effet, ce type d'outils est absent des collections armoricaines depuis le Néolithique moyen I (Guyodo, 2005). Il faut alors regarder du côté de la Normandie sédimentaire, où les tranchets existent dans des contextes du Néolithique moyen II à Banville (Calvados; Kerdivel et Hamon, 2010), à Mondeville (Calvados ; Chancerel et al., 2006) ou à Argentan (Orne; Ghesquière et Marcigny, 2004). D'ailleurs, les tranchets y sont aussi de taille modeste et façonnés sur éclats et par des retouches directes abruptes, parfois envahissantes, seulement sur une face.

L'objet de parure n'est pas datant en lui-même. Nous pouvons toutefois signaler un cas comparable découvert sous le dolmen de Cairon, dans un niveau dont les datages isotopiques sont centrés sur le début du IV millénaire, même si de telles pièces existent en contexte plus ancien (Ghesquière et Marcigny, 2011 : 127-133; Chancerel et al., 2006).

Pour finir, les tessons céramiques décimétriques de la Bourdais 1 se composent d'une anse à ensellement médian, d'un bouton de préhension et d'un bord (fig. 8). Ceux-ci renvoient plutôt au corpus du Néolithique moyen et ne dénotent guère dans sa deuxième étape. 
Figure 8 : Mobilier céramique provenant de la Bourdais I à Saint-Mars-sur-Colmont $(1$ : anse à ensellement médian ; 2 : bouton de préhension ; $3:$ bord). Dessin et DAO : G. Kerdivel.

Figure 8: Ceramic artefacts from la Bourdais I in Saint-Mars-sur-Colmont.

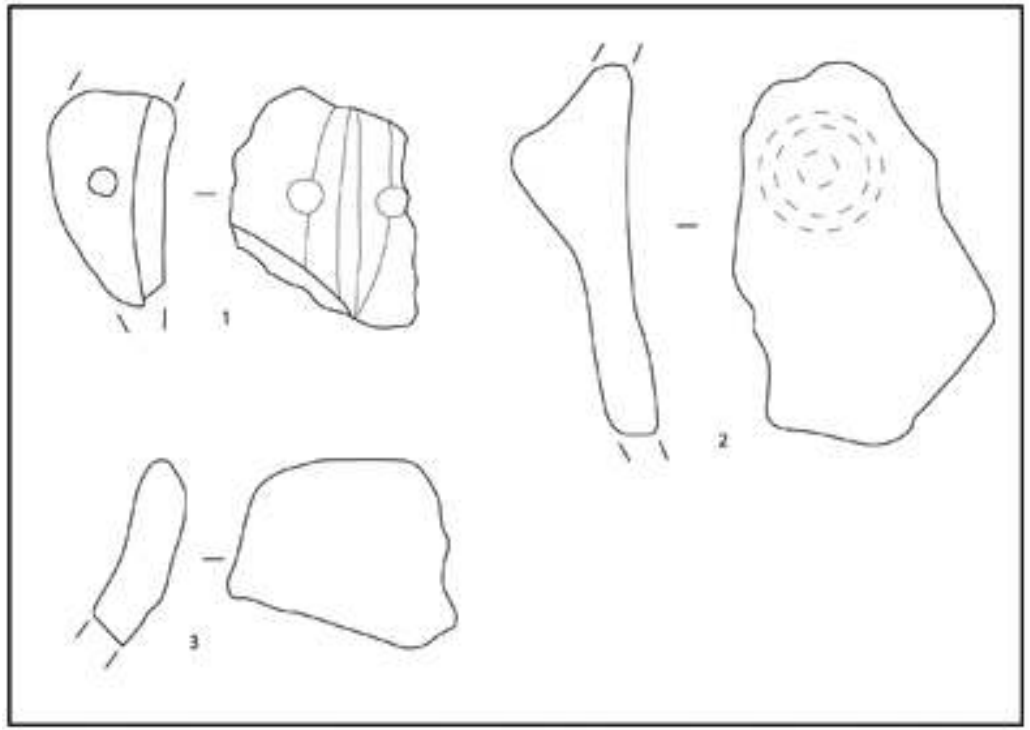

\section{Une acquisition sur place de la dolérite et un façonnage de haches}

Dans les environs immédiats du lieu-dit Beulin, à Saint-Germain-le-Guillaume (fig. 1, $\mathrm{n}^{\circ} 15$; fig. 8) passe un filon de dolérite qui a été exploité par les Néolithiques, comme le suggèrent les résultats d'un sondage récent, confirmant une datation à la fin du Néolithique moyen et/ou au début du Néolithique récent (Kerdivel, 2012 ; Kerdivel et al., 2011). Les affleurements dans ce secteur de la Mayenne sont de très faible puissance, ce qui explique que ce filon ait été exploité à ciel ouvert depuis la surface et non en carrière comme à Plussulien (Côtes-d'Armor ; Le Roux, 1999). Le site livre en surface et à la fouille un grand nombre d'éclats de dolérite, des percuteurs et de rares ébauches.

\section{Synthèse et perspective}

Depuis les découvertes de Saint-Étienne-en-Coglès, la présence d'un Néolithique ancien Villeneuve-Saint-Germain sur le Massif armoricain n'est plus à démontrer (Cassen et al., 1998 ; Marchand et al., 2006 ; Blanchet et al., 2010). Ce n'est donc pas une surprise d'en rencontrer des indices, même ténus, en Mayenne, notamment durant son étape ancienne (Bazougers). Pour l'heure, les indices de bracelets en schiste découverts, appartenant potentiellement au Néolithique ancien, sont essentiellement des objets finis, bien que l'indice à confirmer de Ruillé-Froid-Fonds puisse laisser supposer l'existence de productions locales d'anneaux. Toutefois, la présence d'ébauches n'induit pas pour autant une proximité réelle avec les gîtes de matières premières : une réelle déconnexion peut exister, comme le montre le site de Mondeville (Calvados, Chancerel et al., 2006). D'ailleurs, dans le cas du Néolithique ancien, il ne semble pas qu'une proximité spatiale entre gîte de matières premières lithiques et lieu de consommation ait été un élément déterminant, puisque le recours à de tels matériaux locaux sur le 
Massif armoricain est mineur, excepté pour le façonnage des haches (Cassen et al., 1998 ; Marchand et al., 2006 ; Blanchet et al., 2010). En effet, c'est à cette époque que la fibrolite semble exploitée, ainsi que la dolérite (Pailler, 2007 ; Clément-Sauleau et al., 2010).

31 L'abondance des filons de dolérite dans ce secteur a sûrement été un atout dans l'occupation de la Mayenne, au moins dès le Néolithique moyen, comme semble le montrer une analyse spatiale des gisements de cette période à partir des polissoirs fixes, des gisements du Néolithique moyen et de ceux du Néolithique récent et final. La découverte du gisement de Beulin à Saint-Germain-le-Guillaume vient d'ailleurs conforter cette approche spatiale extra régionale et le modèle de peuplement qui en a été proposé (Kerdivel, 2012b). En outre, cette découverte pose des jalons pour interpréter la place de la Mayenne, une des marges orientales du Massif armoricain, dans les relations socio-économiques de l'Ouest de la France au Néolithique.

Si les données pour le Néolithique ancien sont encore biaisées, puisqu'elles ne concernent que la Normandie sédimentaire (les quelques haches d'Écouché), celles pour le Néolithique moyen méritent d'être développées (fig. 9). Si une exploitation de la dolérite est aujourd'hui indiscutable, celle-ci a pu être disséminée au gré des nombreux filons de la Mancellia, comme autant de petits lieux d'approvisionnement. En outre, si on regarde vers une région dénuée de dolérite comme la plaine d'Argentan (Orne), les découvertes récentes d'éclats dans cette matière attestent de son déplacement. Ainsi, sur le site de l'usine Arma-Maquette à Argentan, un foyer néolithique a été découvert, bien isolé sur ce site mésolithique (Leroy, 1991). Un tesson caréné permet de lui proposer une datation au Néolithique moyen lato sensu (Kerdivel, 2012a). Un mobilier lithique dispersé et mélangé avec les artefacts mésolithiques, notamment un éclat de dolérite, a aussi été rapproché de cette occupation (Ghesquière et Marcigny, 2004). Non loin de là, un éclat et une ébauche de lame de hache en dolérite ont été découverts sur le site Néolithique moyen II du Grand Beaulieu sur la même commune (Ghesquière et Marcigny, 2004). D’autres sont signalés sur l'enceinte de Goulet (Marcigny 2010 : 447). Or, les filons de dolérite reconnus les plus proches sont ceux de l'ouest de l'Orne, à une quarantaine de kilomètres, passant notamment à Saint-Bômer-les-Forges, où un dolmen à couloir est connu (Ménillet et Trautmann, 1987; Chancerel, 1992). Ils appartiennent à cet ensemble de filons de dolérite dont l'exploitation néolithique a été identifiée à $80 \mathrm{~km}$ environ au sud de la plaine d'Argentan. 
Figure 9 : Carte de situation topographique du site d'acquisition de dolérite de Beulin à SaintGermain-le-Guillaume avec vue de la zone excavée à la préhistoire et mobilier en dolérite découvert en surface. DAO et cliché : $G$. Kerdivel.

Figure 9: Topographical map with the site of Beulin in Saint-Germain-le-Guillaume with view from the prehistoric excavated area and surface artefact in dolerite.

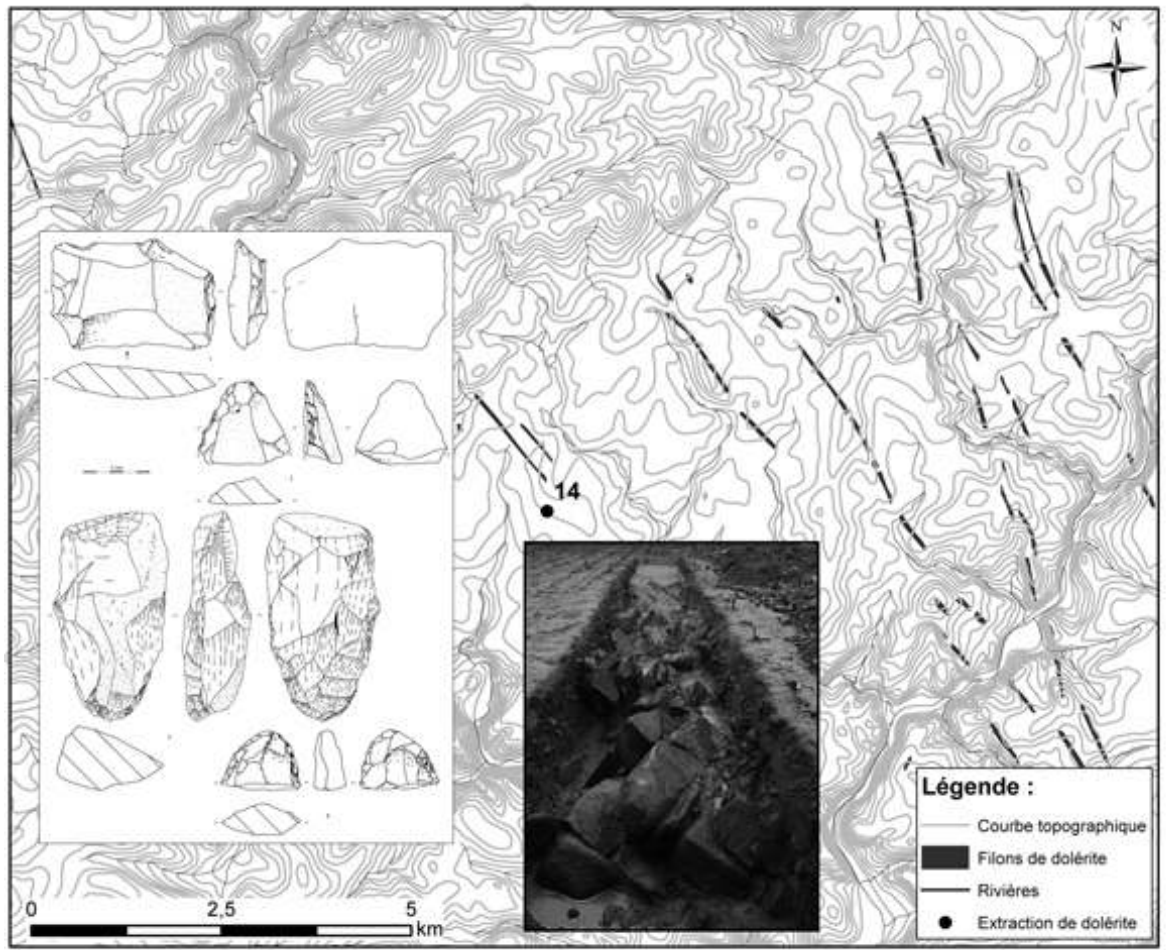


Figure 10 : Carte de synthèse des hypothèses développées dans le texte avec quelques-uns des sites mentionnés

Figure 10: Synthesis map of interpretations discussed in the text with some other sites

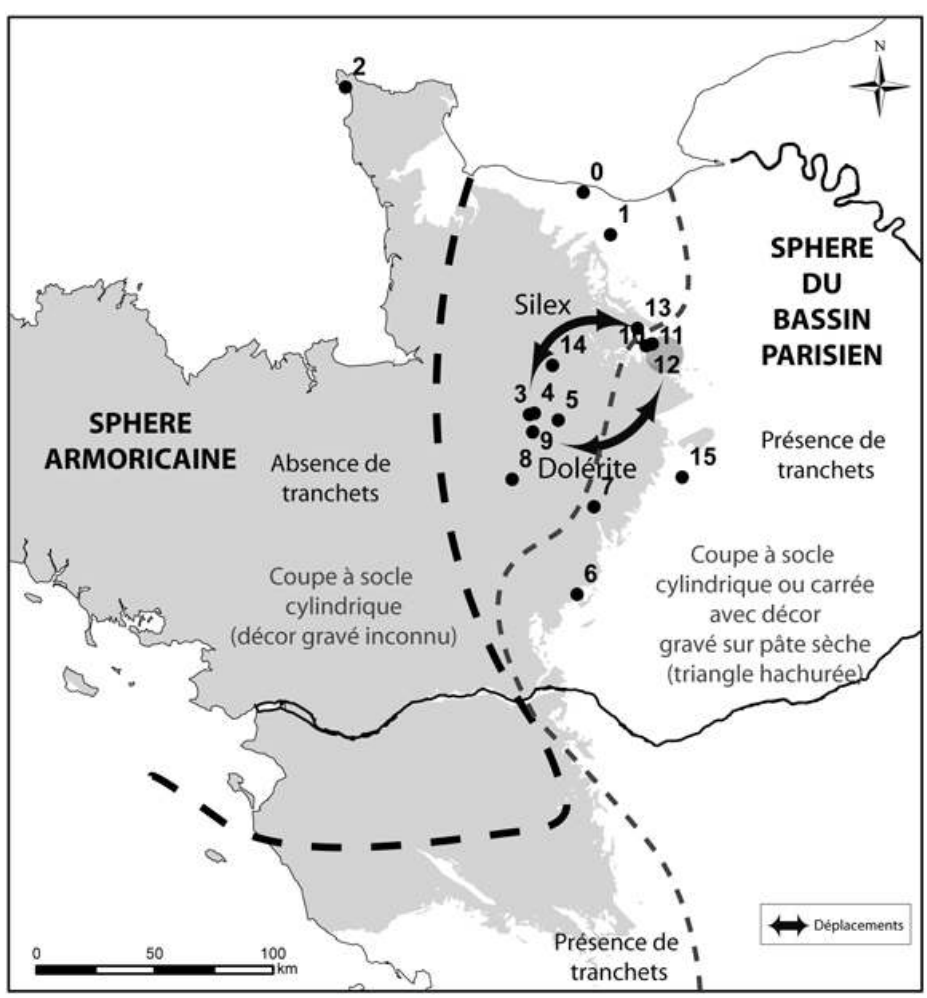

(0 : La Burette à Banville,

Calvados ; 1 : Mondeville sud à Grentheville, Calvados ; 2 : Les Treize Vents à Herqueville, Manche ; 3 Le Val : Couesmes-Vaucé, Mayenne ; 4 : Le Petit Domaine à Couesmes-Vaucé, Mayenne ; 5 : Le Bignon à Lassay-les-Châteaux, Mayenne ; 6 : La Moinerie à Saint-Denis-d'Anjou, Mayenne ; 7 : Les Erves à Sainte-Suzanne ; 8 : Beulin à Saint-Germain-le-Guillaume, Mayenne ; 9 : La Bourdais I à Saint-Mars-surColmont, Mayenne ; 10 : Usine Arma-Maquette à Argentan, Orne ; 11 : Le Grand Beaulieu à Argentan, Orne ; 12 : Goulet, Orne ; 13 : Le Fresne à Rî, Orne ; 14 : Le Creux à Saint-Bômer-les-Forges, Orne ; 15 : Le Parc à Vivoin, Sarthe). CAO et DAO : G. Kerdivel.(0: La Burette in Banville, Calvados; 1: Mondeville sud in Grentheville, Calvados; 2: Les Treize Vents in Herqueville, Manche; 3: Le Val: Couesmes-Vaucé, Mayenne; 4: Le Petit Domaine in Couesmes-Vaucé, Mayenne; 5: Le Bignon in Lassay-les-Châteaux, Mayenne; 6: La Moinerie in Saint-Denis-d'Anjou, Mayenne; 7: Les Erves in Sainte-Suzanne; 8: Beulin in Saint-Germain-le-Guillaume, Mayenne; 9: La Bourdais I in Saint-Mars-sur-Colmont, Mayenne; 10: Usine Arma-Maquette in Argentan, Orne; 11: Le Grand Beaulieu in Argentan, Orne; 12: Goulet, Orne; 13: Le Fresne in Ri, Orne; 14: Le Creux in Saint-Bômer-les-Forges, Orne; 15: Le Parc in Vivoin, Sarthe).

À l'inverse, la présence majoritaire d'un silex provenant de cette région sédimentaire dans des collections mayennaises montre bien le déplacement conjoint de ces deux matériaux. Cela peut être soit la contrepartie d'un système d'échange soit le témoin d'un déplacement logistique de ces populations de la plaine d'Argentan vers ce secteur de la Mayenne. Quoi qu'il en soit, c'est surtout le témoin de contacts entre ces deux secteurs distants (Kerdivel, 2012a) ; d'autant plus que ce silex est largement utilisé dans les collections locales (Ghesquière et Marcigny, 2004: 46). Bien sûr, il n'existe pas encore de preuves comparables à celles de Beulin d'une exploitation de la dolérite dans la région de Couesmes-Vaucé/Saint-Mars-sur-Colmont. Cela implique donc que notre hypothèse repose sur une déconnexion spatiale évidente, relevant surtout de recherches insuffisantes.

Or, les observations de l'équipe de C. Marcigny dans le cadre de l'étude des minières de Ri renforcent les nôtres. Selon ces chercheurs, les collections de surface de la plaine d'Argentan contiennent de nombreuses lames de haches en dolérite, limitant la zone 
d'expansion de la production des haches en silex de la minière de Ri (Marcigny, 2010 : fig. 358). Il existe donc de véritables contacts entre cette partie de la Normandie sédimentaire et le Massif armoricain oriental.

Toutefois, si les matières premières attestent de contacts entre deux régions aux ressources différentes et spécifiques, elles n'en sont pas les seuls témoins. La présence de tranchets façonnés sur éclats est une spécificité des gisements mayennais au regard des collections armoricaines (Guyodo, communication orale). Comme nous l'avons souligné, ces artefacts sont plutôt à rapprocher des assemblages lithiques des Néolithiques de la Normandie sédimentaire, notamment ceux de la plaine d'ArgentanÉcouché (Ghesquière et Marcigny, 2004).

Ainsi, le matériel lithique tend à ancrer le Néolithique mayennais dans une sphère orientale, plus que dans celle du Massif armoricain. C'est aussi le cas de la coupe à socle du dolmen des Erves à Sainte-Suzanne, qui possède un décor de triangles hachurés, réalisé par gravure sur pâte sèche ou après cuisson (Letterlé, 1986) : cette technique de décor est plutôt rare sur le Massif armoricain, de même que le motif (Letterlé, 1986 : 160 ; Hamon, 2003 : 293). Or, ceux-ci sont très fréquents dans le Bassin parisien, comme par exemple à Jonquières, (Oise ; Blanchet et Petit, 1972), à Chassey-le-Camp, (Saône-etLoire ; Thévenot ; 2005) ou aux Châtelliers à Amboise (Indre-et-Loire ; Cordier, 1995). Le motif est aussi connu sur des fragments de coupes à socles à fût cubique découverts à Argentan (Ghesquière et Marcigny, 2004). Cette technique décorative, plus que la morphologie de ce vase, regarde encore plutôt à l'est, comme les indices du plus ancien Néolithique de ce département le laissent aussi à penser. Toutefois, le Néolithique moyen dans la Sarthe et le Maine-et-Loire reste encore très mal connu, incitant à la prudence. Ainsi, les dolmens angevins, bien que centrés sur l'Anjou, sont nombreux dans la Sarthe, ce qui laisse augurer des interactions entre groupes néolithiques plus complexes que ne le propose cet article (Gruet, 1967 ; Kerdivel, 2012a).

Les éléments mobiliers, tant culturels qu'économiques, tendent donc à rapprocher la Mayenne des contextes orientaux plus qu'occidentaux. Or, cet espace oriental des plateaux calcaires jurassiques est celui où le nombre de gisements connus est le plus important au cours des premières étapes du Néolithique, sans qu'il soit possible de n'y voir qu'une conséquence de la recherche (Kerdivel, 2012a et b). Nous avons proposé d'y voir le témoignage d'une certaine facilité de vie, avec des sols plus propices à l'agriculture. Autrement, l'occupation au Néolithique moyen du Massif armoricain apparaît très littorale, tant dans le secteur étudié qu'en Bretagne ; l'intérieur des terres n'y est occupé que sporadiquement. Cette ponctualité de l'occupation intérieure est à mettre selon nous à la charge d'un environnement moins favorable aux cultures et probablement assez fermé mais où sont présentes des matières premières intéressant tout de même les Néolithiques. Un schéma similaire a été aussi proposé pour la Bretagne (Scarre, 2001). Dans ce cas nous y voyons volontiers des occupations courtes, impliquant tout de même une appropriation du territoire que tendraient à montrer les monuments mégalithiques d'une part et des occupations plus longues, voire plus fréquentes de secteurs plus favorables. Ce phénomène pourrait alors témoigner d'un degré de mobilité de ces populations probablement plus fort que ne le laisse paraître la monumentalité de certains mégalithes (Kerdivel, sous presse; Whittle, 2001). 


\section{Conclusion}

Bien qu'il ne s'agisse que de collections de surface, celles-ci présentent un intérêt indéniable au moins à deux niveaux. D'abord, elles permettent de tester le modèle de peuplement proposé par ailleurs, où l'occupation du Massif armoricain serait surtout littorale et ponctuelle dans les terres, et dans ce cas à relier à l'exploitation de roches particulières telles que la dolérite. Toutefois, cette occupation spécifique semble évoluer vers la fin de la période, sous l'influence probable d'une régulière croissance démographique dont témoigne indirectement l'abondance d'allées couvertes et de sépultures à entrée latérale datées de cette phase du Néolithique (Kerdivel, 2012a). Ensuite, et de manière complémentaire, elles offrent l'occasion de replacer la Mayenne dans les réseaux d'échange en vigueur dans l'Ouest de la France et dans les aires culturelles dominantes. Ainsi, si la néolithisation de ce secteur nécessite de nouveaux travaux, peut-être au travers des bracelets en schiste pour lesquels des ateliers semblent exister, ce sont les données pour le Néolithique moyen qui sont les plus novaterice En effet, la mise en évidence durant cette période d'un autre réseau d'approvisionnement en silex, celui d'Écouché, est un élément essentiel à plus d'un titre. D'abord, elle témoigne d'un jeu entre des interactions locales, occidentales ou orientales perceptible au niveau des matières premières comme du panel de l'outillage néolithique où la Mayenne, géologiquement armoricaine semble toutefois être ancrée dans une sphère orientale, c'est-à-dire plutôt à relier aux groupes humains du Bassin parisien et à leurs traditions. Par ailleurs, elle offre des arguments allant dans le sens d'un degré de mobilité de ces populations probablement plus important qu'il n'y paraît au premier abord. Il reste toutefois à approfondir ces observations par l'étude des gisements et du mobilier récolté dans des contextes plus fiables et à explorer le ou les liens que ce département ne doit pas manquer d'entretenir avec le reste du Maine et de l'Anjou.

Je souhaite remercier pour leurs précieux conseils et commentaires: MM. L. Laporte (CNRS, Rennes), G. Marchand (CNRS, Rennes), C. Marcigny (Inrap, Caen), ainsi que les attentifs relecteurs J.-N. Guyodo (MCF, Nantes) et C. Billard (MCC). Un grand merci à B. Bodinier (GRAM) qui s'est chargé de la correction orthographique.

BIBLIOGRAPHIE

AVIGNON L., 1976 - «Les sépultures mégalithiques de la région d'Evron », Bulletin de Mayenne-

Sciences, p. 72-81.

BAILLOUD G., CORDIER G., GRUET M. et POULAIN T., 1987 - « Le Néolithique ancien et moyen de la vallée de la Brisse (Loir-et-Cher) », Revue archéologique du Centre de la France, 26, p. 117-163.

BLANCHET J.-C. ET PETIT M., 1972 - «L'habitat néolithique de Jonquières (Oise). Premiers résultats », Bulletin de la Société préhistorique française, 69, p. 389-407. 
BLANCHET S., FORRE P., FROMONT N., HAMON C. et HAMON G., 2010 - « Un habitat du Néolithique ancien à Betton « Pluvignon » (Ille-et-Vilaine). Présentation synthétique des premiers résultats », in BILLARD C. et LEGRIS M. (dir.) - Premiers Néolithiques de l'Ouest. Cultures, réseaux, échanges des premiers néolithiques à leur expansion. Colloque interrégional sur le Néolithique Le Havre 2007, Rennes, PUR, (Archéologie et Culture), p. 15-39.

BOUILLON R., 1984 - « Les Temps préhistoriques », in SALBERT J., La Mayenne des origines à nos jours, Saint-Jean-d'Angély, Bordessoules, (coll. Hexagone), p. 28-43.

BOUILLON R., 1989 - « La sépulture mégalithique à entrée latérale du Petit-Vieux-Sou à Brécé (Mayenne) », Revue archéologique de l'Ouest, 6, p. 51-70.

BOUILLON R., 1998 - «L'allée couverte de la Cote 197, à Vautorte, en forêt de Mayenne. Fouille et restauration d'une sépulture mégalithique du Néolithique final », La Mayenne: Archéologie, Histoire, 21, p. 5-48.

CASSEN S., AUDREN C., HINGUANT S., LANNUZEL G. et MARCHAND G., 1998 - « L'habitat Villeneuve-SaintGermain du Haut-Mée (Saint-Étienne-en-Coglès, Ille-et-Vilaine) », Bulletin de la Société préhistorique française, 95, p.41-75.

CHANCEREL A., 1992 - « Les sépultures mégalithiques du département de l'Orne. Un bilan bibliographique », in FICHET DE CLAIRFONTAINE F. (dir.), Archéologie dans l'Orne 1987-1992, Société historique et archéologique, 61, p. 52-77.

CHANCEREL A., MARCIGNY C., GHESQUIÈRE E. et VERRON G., 1996 - « Le site Néolithique Moyen II des « Treize Vents » à Herqueville (Manche) », Bulletin de la Société préhistorique française, 93, p. 241-248.

CHANCEREL A., MARCIGNY C. et GHESQUIÈRE E. (dir.), 2006 - Le plateau de Mondeville (Calvados) du Néolithique à l'âge du Bronze, Paris, Éditions de la Maison des Sciences de l'Homme, (Document d'Archéologie française, 99), $205 \mathrm{p}$.

CLEMENT-SAULEAU S., FROMONT N., GHESQUIÈRE E., GIAZZON D. et MARCIGNY C., 2010 - « Écouché. Carrière MEAC », Bilan Scientifique de Basse-Normandie 2008, Caen, SRA, p. 101.

COLLECTIF, 1986 - Premiers agriculteurs de la Mayenne. Recherches Récentes sur le Néolithique 1978-1986, Nantes, Association d'études préhistoriques et historiques des Pays-de-la-Loire, $41 \mathrm{p}$.

CORDIER G., 1995 - « Le site chasséen du plateau des Châtelliers à Amboise (Indre-et-Loire).

Découverte et fouilles A. et S. Hogstrom (1954-1957) », Revue archéologique du Centre de la France, 34, p. 109-155.

DRON J.-L., GERMAIN-VALLÉE C., CLÉMENT-SAULEAU S., GÂCHE D., CHARRAUD F. et FROMONT N., 2010 - « La Bruyère du Hamel à Condé-sur-Ifs Calvados). Un site entre Néolithique ancien et Néolithique moyen ", in BILLARD C. et LEGRIS M. (dir.) - Premiers Néolithiques de l'Ouest. Cultures, réseaux, échanges des premiers néolithiques à leur expansion. Colloque interrégional sur le Néolithique Le Havre 2007, Rennes, Presses universitaires de Rennes, (Archéologie \& Culture), p. 163-179.

DUBOULOZ J., 2003 - «Datation absolue du premier Néolithique du Bassin parisien : complément et relecture des données RRBP et VSG », Bulletin de la Société Préhistorique Française, 100, p. 671-689. FROMONT N., 2008 - « Les anneaux du Néolithique bas-normand et du Nord-Sarthe : production, circulation et territoires », Bulletin de la Société préhistorique française, 105, p. 55-86.

FROMONT N., HUARD J., KERDIVEL G., LERIVRAIN R., MENS E., QUERRE G. avec la collaboration de BODINIER B., M. et G., FOUCHER G., LECOMTE V., LENORMAND A. et F., PRIOUX F., BERNARD-GRIFFITHS P. et J., AVRIL J.-Y., THARRAULT J. et CHOMIENNE N., 2008 - Rapport de prospection thématique. Sites néolithiques d'acquisition et de transformation de la dolérite en Mayenne. Prospections pédestres sur les communes d'Andouillé, Saint- 
Germain-d'Anxure, La Bigottière, Saint-Germain-le-Guillaume et Alexain. Résultats des années 2007 et 2008, Nantes, SRA, $24 \mathrm{p}$.

GEORGES E. et KERDIVEL G. avec la collaboration de GUYODO J.-N., HAMON G., LENORMAND A. et MENS E., 2012 « Habitat et site d'extraction de silex au début du Néolithique moyen. Les sites de la Croix-SainteAnne à Juigné-sur-Sarthe et du Camp de César à Vion (Sarthe) », in DE LABRIFFE P.-A. et THIRAULT E. (dir.), Produire des haches au Néolithique : de la matière première à l'abandon. Table-Ronde de la SPF des 16 et 17 mars 2007. Saint-Germain-en-Laye, séance de la SPF, p. 173-190.

GILBERT M., 1964 - Menhirs et dolmens dans le nord-est de la Bretagne, Guernesey, Guernesey Press Co. GHESQUIÈRE E., MARCIGNY C. et DRON J.-L., 1998 - « Le débitage lithique au Cerny en Basse-Normandie », Internéo, 2, p. 57-63.

GHESQUIÈRE E. et MARCIGNY C. (dir.) avec les contributions de AUBRY B., CLÉMENT-SAULEAU S., DIETSCHSELLAMI M.-F., DELOZE V., HAMON G., QUERRE G. et RENAULT V. 2003 - « L'habitat néolithique moyen I de Vivoin « Le Parc» (Sarthe) », Bulletin de la Société préhistorique française, 100, p. 533-573.

GHESQUIÈRE E. et MARCIGNY C., 2004 - Carrières de calcaire Néolithique moyen II à Argentan (Orne), Internéo, 5, p. 45-62.

GHESQUIÈRE E. et MARCIGNY C. (dir.), 2011 - Cairon. Vivre et mourir au Néolithique. La Pierre Tourneresse en Calvados, Rennes, Presses Universitaires de Rennes, (Archéologie \& Culture), 199 p.

GRUET M., 1967 - Inventaire des mégalithes de la France 2 - Maine-et-Loire, Paris, CNRS ( $1^{\mathrm{er}}$ suppl. à Gallia Préhistoire), 345 p.

GUYODO J.-N., 2001a - Les assemblages lithiques des groupes néolithiques sur le Massif armoricain et ses marges, Thèse de Doctorat, Université de Rennes 1, 466 p.

GUYODO J.-N. avec la collaboration de NOSLIER A., MADIOUX P. et BIZIEN-JAGLIN C., 2001b - « L'assemblage lithique du site néolithique moyen II de Lillemer (Ille-et-Vilaine) », Bulletin de la Société préhistorique française, 98, p. 647-662.

GUYODO J.-N., 2003 - « Acquisition et circulation des matières premières au Néolithique dans l'ouest de la France ", in COLLECTIF, « Les matières premières lithiques en préhistoire. Table-ronde internationale organisée à Aurillac (Cantal), du 20 au 22 juin 2002 », Préhistoire du Sud-Ouest, 5, p. 185-197.

GUYODO J.-N., 2005 - « Les assemblages lithiques de la fin du Néolithique ancien et du Néolithique moyen sur le Massif armoricain et ses marges », in MARCHAND G. et TRESSET A. (dir.) - Unité et diversité des processus de Néolithisation sur la façade atlantique de l'Europe (VII et IVe millénaires avant J.-C.). Table ronde de Nantes 26-27 avril 2002, Paris, Société Préhistorique Française, (Mémoires, 36) p. 213-224.

HAMON G., 2003 - Les productions céramiques au Néolithique ancien et moyen dans le nord-ouest de la France, Thèse de Doctorat, Université de Rennes 1, 2 tomes, 329 p., 121 pl.

JousSAUME R. et PAUTREAU J.-P., 1990 - La Préhistoire du Poitou, Rennes, Éditions Ouest-France, (Coll. Université), $599 \mathrm{p}$.

JOUSSAUME R. et LAPORTE L., 2006 - « Monuments funéraires néolithiques dans l'ouest de la France », in JOUSSAUME R., LAPORTE L. et SCARRE C. (dir.), Origine et développement du mégalithisme de l'ouest de l'Europe. Actes du Colloque International, 26-30 octobre 2002, Bougon (France), Niort, Conseil général des Deux-Sèvres, vol. 1, p. 319-343. 
JOUSSAUME R., CREDOT R. et GIRAUD C., 2008 - « Le dolmen des Goudours à Folles (Haute-Vienne) et les dolmens à chambre axiale allongée dans le Centre-Ouest de la France », Préhistoire du Sud Ouest, 16 , p. 3-54.

KERDIVEL G., 2012A - Occupation de l'espace et gestion des ressources à l'interface entre massifs primaires et bassins secondaires et tertiaires. L'exemple du Massif armoricain et de ses marges au Néolithique, Oxford, Archaeopress, (BAR International Series, 2383), 362 p.

KERDIVEL G., 2012B - « Rôle des roches tenaces dans la distribution spatiale des gisements du Néolithique moyen au Néolithique récent et final à l'interface Massif armoricain/Bassins parisien et aquitain », in MARCHAND G. et QUERRE G., (dir.), Roches et Sociétés en Préhistoire entre massifs cristallins et bassins sédimentaires, Rennes, PUR, (Archéologie \& Culture).

KERDIVEL G., QUERRE G., TORRADO ALONSO A. et LE GRAM, 2011 - « Exploitation de la dolérite dans le nordouest de la Mayenne au Néolithique : données préliminaires », Bulletin de la Société géologique et minéralogique de Bretagne, $\mathrm{n}^{\circ}$ 9, Série D, p. 35-51.

KERDIVEL G. et HAMON G. avec la collaboration de BARGE J., BOHARD B., DESLOGES J. ET LEPAUMIER H., 2010 «Un site du Néolithique moyen, du Néolithique final et de l'âge du Fer à la Burette à Banville (Calvados) : Présentation liminaire », in BILLARD c. et LEGRIS M. (dir.), Premiers Néolithiques de l'Ouest. Cultures, réseaux, échanges des premiers néolithiques à leur expansion. Colloque interrégional sur le Néolithique Le Havre 2007, Rennes, Presses Universitaires de Rennes, (Archéologie \& Culture), p. 211-235.

LARGE J.-M. et BIROCHEAU P. (dir.) avec la collaboration de CROS J.-P. et DUDAY H., 2004 - Les Châtelliers du Vieil-Auzay (Vendée). Une archéologie d'un site exceptionnel de la Préhistoire récente, La Roche-sur-Yon, Groupe Vendéen d'études préhistoriques, $686 \mathrm{p}$.

LASSEUR E., LECLERC F. et CLIQUET D., 2005 - « Accessibilité, acquisition et utilisation de la matière première : Etude gîtologique de deux gisements du Paléolithique moyen, la vallée de la Seulles (Calvados) et le site de Saint-Brice-sous-Rânes (Orne) ", in MOLINES N., MONCEL M.-H. et MONNIER J.-L. (éd.), Les premiers peuplements en Europe, Oxford, Archaeopress, (BAR, International Series, 1364), p. 409-417.

LE FIZELIER J., 1878 - Etudes sur la géographie ancienne du Bas-Maine. Arvii et Diablintes, Tours, P. Bouserez.

LE ROUX C., 1999 - L'outillage de pierre polie en métadolérite du type A. Les ateliers de Plussulien (Côtesd'Armor) : production et diffusion au Néolithique dans la France de l'ouest et au-delà, Rennes, Association des Travaux du Laboratoire d'Anthropologie, (Travaux du Laboratoire, 43), 244 p.

LEDRU A., 1911 - Répertoire des Monuments et objets anciens préhistoriques, gallo-romains, mérovingiens et carolingiens existant ou trouvés dans les départements de la Sarthe et la Mayenne, Archives historiques du Maine, $431 \mathrm{p}$.

LEROY D. avec la collaboration de GHESQUIERE E. et HERARD B., 1991 - « Le site mésolithique d'ArmaMaquette à Argentan, Orne », Revue archéologique de l'Ouest, 8, p. 25-46.

LETTERLE F., 1986 - «Le dolmen des Erves à Sainte-Suzanne et ses implications chronologiques », in COLLECTIF, Actes du 10e colloque interrégional sur le Néolithique à Caen de 1983, Revue Archéologique de l'Ouest, (supplément $\mathrm{n}^{\circ}$ 1), p. 149-165.

MARCHAND G., PAILLER Y. et TOURNAY G., 2006 - « Carrément à l'Ouest ! Indices du Villeneuve-SaintGermain au centre de la Bretagne (le Dillien à Cléguérec et Bellevue à Neulliac ; Morbihan) », Bulletin de la Société préhistorique française, 103, p. 519-533. 
MARCIGNY C. (dir.), 2010 - Ri et Ronai (Orne), «Le Fresne ». Une minière de silex du Néolithique. Rapport final d'opération, Inrap Grand Ouest, Vol. 1, 543 p., inédit.

MARCIGNY C., GHESQUIÈRE E. et RICHE C. avec la collaboration de MALLET N., 2007 - « Analyse du mobilier funéraire du mégalithe du « Château » à Angers (Maine-et-Loire) : présence d'outils en silex du Grand-Pressigny ", Bulletin de la Société des amis du musée de préhistoire du Grand-Pressigny, 58, p. 19-30.

MARCIGNY C., GHESQUIÈRE E., GIAZZON D., TSOBGOU-AHOUPE R., CHARRAUD F., JUHEL L. et GIAZON S., 2011 - « The flint mine of Ri « Le Fresne » », in CAPOTE M., CONSUEGRA S., DIAZ-DEL-RIO P. et TERRADAS X. (éd), Proceedings of the $2^{\text {nd }}$ International Conference of UISPP Commission on Flint Mining in Pre-and Protohistoric Times (Madrid, 14-17 October 2009), Oxford, Archaeopress, (BAR International Series, 2260), p. 67-75.

MENILLET F. et TRAUTMANN F. avec la collaboration de FOURNIGUET J., JANJOU D., LANGEVIN C., LAUTRIDOU J.-P., QUERIERE P. (DE LA), VAUTRELLE C. et VERRON G., 1987 - Notice explicative de la Carte géologique de la France à 1 / 50 000. Mortain, Orléans, BRGM, 70 p. (+ carte).

MOREAU E., 1880 - «Études préhistoriques sur les environs d'Ernée. Polissoir de la Bertellière. Moitié d'anneau en pierre trouvée à Ernée », Bulletin de la Société historique et archéologique de Mayenne, 1, p. 108.

MOREAU E., 1889 - « Disque de silex foré trouvé à Hardanges », Bulletin de la Commission historique et archéologique de la Mayenne, 1, p. 210-213.

PAILLER Y., 2007 - Des dernières industries à trapèzes à l'affirmation du Néolithique en Bretagne occidentale (5500-3500 av. J.-C.), Oxford, Archaeopress, (BAR International Series, 1648), 340 p.

PRAUD I. (dir.), 2009 - Le Néolithique ancien dans la basse vallée de la Marne : un site Villeneuve-SaintGermain producteur de lames en silex tertiaire à Ocquerre «La Rocluche » (Seine-et-Marne), Paris, Société préhistorique française, (travaux, 9), $141 \mathrm{p}$.

SCARRE C., 2001 - « Modeling Prehistoric Populations: The Case of Neolithic Brittany », Journal of Anthropological Archaeology, 20, p. 285-313.

THEVENOT J., 2005 - Le Camp de Chassey (Chassey-le-Camp, Saône-et-Loire) : les niveaux néolithiques du rempart de " la Redoute », Dijon, Société archéologique de l'Est, (supplément à la Revue archéologique de l'Est), $464 \mathrm{p}$.

TINEVEZ J.-Y., LORHO T. et QUESNEL L., 2006 - « Vestiges d'habitat du Néolithique moyen I à Quimper, Kervouyec Nevez », Internéo, 6, p. 67-76.

VALAIS A., MEURET J.-C., NAULEAU J.-F. avec la collaboration de EDIN F., HAMON G. et MUTTARELLI E., 2008 « Le Clos-Henry : une ferme de la Tène finale à Château-Gontier (Mayenne) », Revue archéologique de l'Ouest, 25, p. 139-161.

VERRON G., 2000 - Préhistoire de la Normandie, Rennes, Éditions Ouest-France, (Coll. Université), 364 p. VERNHET Y., DORE F., LAUTRIDOU J.-P., TALBO H., VERRON G., DHELLEMES R., ENOUF C., 1995 - Notice explicative de la Carte Géologique de la France au 1/50 000. Domfront, BRGM, Orléans, 106 p. + carte.

WHITTLE A., 2001 - « From mobility to sedentism: change by degrees », in KERTÉSZ R. and MAKKAY J. (dir.), From the Mesolithic to the Neolithic. Proceedings of the International Archaeological Conference held in the Damjanich Museum of Szolnok, September 22-27, 1996, vol. 2, Budapest, Archaeolingua Alapitvany, p. 47-461. 


\section{NOTES}

1. Le mobilier est conservé au dépôt archéologique départemental de Mayenne à Jublains; nous remercions E. Mare (Inrap) de nous avoir autorisés à publier ses trouvailles.

2. La détermination des matériaux schisteux est l'œuvre de N. Fromont (Inrap), que nous remercions.

3. Nous tenons à remercier G. Foucher, V. Lecomte et R. Lerivrain de nous avoir permis d'accéder à leurs collections.

\section{RÉSUMÉS}

Les phases ancienne et moyenne du Néolithique en Mayenne ont longtemps été méconnues, la période ayant été, pendant longtemps, surtout représentée par les monuments de la fin du Néolithique, fouillés par R. Bouillon. Pourtant, des découvertes de surface anciennes et une fouille récente attestent de la présence d'un Néolithique ancien ("casse-tête», anneaux de pierre, assemblage lithique et céramique du Villeneuve-Saint-Germain). De même, le Néolithique moyen était connu par un seul dolmen : celui des Erves à Sainte-Suzanne. Nous présentons ici deux séries de surface inédites, témoignant d'occupations domestiques du Néolithique moyen, qui montrent un emploi important du silex de la région d'Écouché et une présence originale de tranchets sur éclat, encore peu signalée sur le Massif armoricain. Par ailleurs, l'exploitation de la dolérite au Néolithique, dont de nombreux filons existent en Mayenne, est maintenant attestée sur le site de Beulin à Saint-Germain-le-Guillaume. Finalement, les données disponibles actuellement montrent des contacts étroits entre les Néolithiques de la Normandie et ceux de Mayenne, ce qui permet de mieux replacer ce département dans son contexte local et régional, ainsi que de tester un modèle de peuplement récemment proposé qui interroge le degré de mobilité des populations.

During a long time, the Early and Middle Neolithic in Mayenne were few known, the period was represented by Recent Neolithic monuments, excavated by R. Bouillon.But ancient surface discoveries and one recent excavation certify one Early Neolithic presence (stone-ring, " cassetête ", lithic and ceramic of the Villeneuve-Saint-Germain culture). The Middle Neolithic was known by by one single dolmen: Les Erves in Sainte-Suzanne. The domestic settlements of this stage were unknown before the presentation in this paper of two recent surface scatters. They show the important use of flint from the Ecouchés area (Orne, Normandy) and the particular presence of tranchet on flake, away from the Armorican massif. In other way, the dolerite is exploited during the Neolithic. Finally, the available data show contacts between the Neolithic of Normandy and those of Mayenne, rather than those of the Armorican massif (Britanny), what allows to replace better this department in its local and regional context as well as to test a recent model of populating, which questions the degree of mobility of these populations. 
INDEX

Keywords : Neolithic, Armorican massif, Mayenne, Normandy, flint, dolérite, Écouché

Mots-clés : massif armoricain, Mayenne, Normandie, silex, dolérite, Écouché, Néolithique

\section{AUTEUR}

GWENOLÉ KERDIVEL

Département Histoire de l'Art et Archéologie et laboratoire LARA - Université de Nantes Chemin de la Censive-du-Tertre - BP 81227 - 44312 NANTES Cedex 3. gwenole.kerdivel@univnantes.fr. 